\title{
Correlation between the anti-Müllerian hormone and endovaginal ultrasound in the predictivity of polycystic ovary syndrome at Chracerh
}

\author{
Noa Ndoua C. C. ${ }^{1 *}$, Ayissi Ngah G. ${ }^{4}$, Mve Koh V. ${ }^{3}$, Belinga E. ${ }^{2}$, Kemfang J. D. ${ }^{2}$, Kasia J. M. ${ }^{2}$ \\ ${ }^{1}$ Department of Medicine and Biomedical Sciences, University of Yaounde, Cameroon \\ ${ }^{2}$ Department of Obstetrics and Gynecology, Hospital Research and Application Centre for Endoscopic Surgery and \\ Human Research, Cameroon \\ ${ }^{3}$ Department of Obstetrics and Gynecology, University Hospital of Yaounde, Cameroon \\ ${ }^{4}$ Department of Medicine and Pharmaceutical Sciences, University of Douala, Cameroon
}

Received: 01 October 2017

Accepted: 31 October 2017

\section{*Correspondence:}

Dr. Noa Ndoua C. C.,

E-mail: claudenoa@yahoo.co.uk

Copyright: $\odot$ the author(s), publisher and licensee Medip Academy. This is an open-access article distributed under the terms of the Creative Commons Attribution Non-Commercial License, which permits unrestricted non-commercial use, distribution, and reproduction in any medium, provided the original work is properly cited.

\section{ABSTRACT}

Background: Polycystic ovary syndrome (PCOS) is a heterogeneous syndrome posing diagnostic problems in current practice, because of the cumbersomeness associated with the application of the criteria that define it; giving rise to over or under diagnosis of it. Anti-Müllerian hormone (AMH) is an effective alternative in this case, being a stable, reproducible and non-operator dependent marker to diagnose PCOS due to the link that binds it to the multiple preantral follicles in the ovaries of PCOS patients. The aim of this study was to determine the threshold value of AMH required to define PCOS in our African population, by revealing the correlation with antral follicles count (AFC) on endovaginal ultrasound.

Methods: We carried out a comparative cross-sectional study, with retrospective data collection in 23 infertile patients diagnosed with PCOS according to the Rotterdam 2003 criteria, and 23 non-PCOS infertile controls having performed the AMH test using the Immunotech technique at CHRACERH. Endovaginal ultrasound (U/S) was performed using a 7.5 mega Hertz $(\mathrm{MHz})$ transvaginal transducer by different qualified operators (radiologists, gynecologists). The comparison of the two independent groups (PCOS and non-PCOS) was performed by the Student t-test; correlations between AMH, age, AFC and ovarian volume obtained by the Pearson test; and the diagnostic power of AMH test in PCOS was evaluated by receiver operating characteristic curves (ROC).

Results: AMH was approximately twice as high in PCOS compared to controls $(6.09$ versus $3.80, \mathrm{P}<0.001)$ and was inversely correlated with age $(\mathrm{r}=-0.301 ; \mathrm{P}<0.05)$; significantly correlated antral follicle count $(\mathrm{R}=0.85, \mathrm{P}<0.0001)$ and ovarian volume $(\mathrm{r}=0.625, \mathrm{P}<0.0001)$. ROC analysis revealed that the AMH test was very informative for the diagnosis of PCOS with an area under the curve (AUC) at $0.90(0.81-0.99 ; 95 \% \mathrm{CI})$; and the threshold value given by the farthest point of the diagonal representing the nil contribution test was $4.40 \mathrm{ng} / \mathrm{L}$, and predicted PCOS with a sensitivity of $96 \%$ and a specificity of $70 \%$.

Conclusions: AMH is a predictive marker for PCOS. It is highly correlated with AFC and ovarian volume and appears to decrease with age. It offers good diagnostic performance in PCOS, with a threshold value of $4.40 \mathrm{ng} / \mathrm{L}$ for a sensitivity of $96 \%$ and specificity of $70 \%$.

Keywords: AMH, Follicules antraux, PCOS

\section{INTRODUCTION}

Polycystic ovary syndrome is the most common cause of anovulatory infertility and endocrine abnormalities in women of childbearing age. ${ }^{1}$ It is an heterogeneous syndrome which may vary from one patient to another, and which combines when fully expressed cutaneous hyperandrogenism, menstrual irregularity and polycystic 
ovaries on ultrasound. ${ }^{2}$ As the name suggests, it is a syndrome, not a disease so there is no test for its definitive diagnosis but rather sets of diagnostic criteria established for it.

Three groups of diagnostic criteria exist in the literature to define it, including the National Institute of Health criteria of 1990, which combine clinical or biological hyperandrogenism and menstrual irregularity; those of Rotterdam 2003 and the revised criteria of NIH 2012 which add to the previous, the definition of polycystic ovaries aspect on endovaginal ultrasound; and the Androgen Excess Society criteria (AES), which differ from the latter in that, they don't integrate women with menstrual irregularity and polycystic aspect of the ovaries, without evidence of androgens excess in the diagnosis of PCOS..$^{3-5}$ As a result, the prevalence of PCOS varies according to the criteria used to diagnose it. It is 6-10\% using the NIH 1990 criteria and 14-17\% using the Rotterdam 2003 criteria for women of childbearing age. ${ }^{6,7}$ It is also likely that many women in the general population are either over-diagnosed or under-diagnosed, due to the heterogeneity of the clinical presentation and the criteria defining PCOS. This underscores the importance of identifying a standardized diagnostic tool with minimal inter-observer variation.

Anti-Müllerian hormone (AMH), a glycoprotein belonging to the TGF- $\beta$ family (Transformation Growth Factor), then appears as a potentially attractive alternative. Indeed, the AMH produced by the small preantral follicles is a stable product, the measurement of which is not subjective compared to the endovaginal ultrasound, which is an operator-dependent technique. Moreover, the correlation between AMH and the number of antral follicles is established. ${ }^{8}$ It is also correlated with the biological markers of hyperandrogenism (testosterone and androstenedione), oligomenorrhea and ovarian volume. Hence, AMH could be a potential biological marker in the diagnosis of PCOS, bearing in mind the limits of its sensitivity and specificity. Due to the racial and environmental variability of PCOS, we sought to determine for the first time in our setting the predictive AMH threshold for PCOS in an African population and to highlight the correlation between $\mathrm{AMH}$ and findings at Endovaginal ultrasound.

\section{METHODS}

We carried out a cross-sectional, retrospective and analytic study, over 5 years, from the $1^{\text {st }}$ May 2012 to $31^{\text {st }}$ December 2016. The study was carried out over a five months period, from the $1^{\text {st }}$ January to $1^{\text {st }}$ May 2017, at the Hospital Center for Research and Application in Endoscopic Surgery and Human Reproduction (CHRACERH) in Yaounde-Cameroon.

Data (age, body mass index, Rotterdam criteria for the diagnosis of PCOS, AMH levels, antral follicles count and ovarian volume) was collected from the records of infertile PCOS patients, diagnosed by the Rotterdam 2003 criteria and infertile non-PCOS patients, admitted for in vitro fecundation (IVF) who had performed an AMH test according to the Immunotech assay technique (IOT), and endovaginal ultrasound during that IVF session at CHRACERH. An authorization was obtained beforehand from the CHRACERH ethics committee.

\section{Patients}

\section{Controls}

Control population consisted of the records of 23 women under 38 years who were admitted in our hospital for in vitro fertilization because of tubal and/or male infertility. They had an AMH test at least $\geq 2 \mathrm{ng} / \mathrm{L}$ according to the IOT technique, and an endovaginal ultrasound performed at CHRACERH using a 7.5 MHZ transvaginal transducer by different qualified operators (radiologists, gynecologists). The non-inclusion criteria were records of infertile non-PCOS patients, admitted in IVF with an AMH level less than $2 \mathrm{ng} / \mathrm{L}$, with ovarian failure or early menopause.

\section{Patients with PCOS}

Twenty-three records of women under 38 years were enrolled in this study. According to the Rotterdam criteria, the diagnosis of PCOS was based on the association of at least two of the three following criteria:

- Ovulatory disturbance, mainly oligomenorrhea or amenorrhea

- Hyperandrogenism (HA) as defined either clinically by hirsutism (modified Ferriman and Gallwey score $>6$ ), or severe acne/seborrhea, and/or biologically by a testosterone serum level greater than $0.7 \mathrm{ng} / \mathrm{ml}$ and/or $\Delta 4$-androstenedione greater than $2.2 \mathrm{ng} / \mathrm{ml}$

- More than 12 follicles in the 2 to $9 \mathrm{~mm}$ range in each ovary at U/S and/or an ovarian volume higher than $10 \mathrm{ml}^{3}$

U/S examination has been performed with a $7.5-\mathrm{MHz}$ transvaginal transducer by different qualified operators (radiologists, gynecologists). and the AMH assay was performed according to the IOT assay technique at CHRACERH.

\section{Statistical analysis}

Receiver operating characteristic (ROC) curves were constructed to examine the diagnostic performance of $\mathrm{AMH}$ test, that is its capacity to discriminate between the controls and patients with PCOS. Sensitivity (y-axis) against [1-specificity (x-axis)] was plotted at each threshold level, and the area under the curve (AUC) was computed by the parametric method by the SPSS 20 software. AUC represents the probability of correctly identifying controls and patients with PCOS. A value of 0.5 means that the test result is no better than chance. 
Comparisons of two independent groups (PCOS and nonPCOS patients) were performed using the Student $t$ test for normally distributed variables and the correlations between AMH, age, AFC and ovarian volume obtained by the Pearson test. All statistical analyses were performed using SPSS 20 Software (SPSS Inc., Chicago, IL).

\section{RESULTS}

\section{Age, weight and $\mathrm{BMI}$}

The mean age, weight and BMI of PCOS patients and non-PCOS controls are summarized in Table 1 . No statistically significant differences were found between these parameters in the two groups.

Table 1: Distribution of the study population according to some biophysical parameters.

\begin{tabular}{|llll|} 
Parameters & $\begin{array}{l}\mathrm{PCOS}+ \\
(\mathrm{n}=23)\end{array}$ & $\begin{array}{c}\text { PCOS }- \\
(\mathrm{n}=23)\end{array}$ & P value \\
\hline Age & $32.43 \pm 4.04$ & $34.30 \pm 3.00$ & 0.083 \\
\hline Weight & $73.95 \pm 13.17$ & $70.93 \pm 9.80$ & 0.382 \\
\hline BMI & $27.20 \pm 4.90$ & $26.80 \pm 4.62$ & 0.778 \\
\hline
\end{tabular}

Mean values of $A M H, A F C$ and ovarian volumes in PCOS versus controls

The mean serum AMH level was 2-fold higher in PCOS patients than in controls $(\mathrm{P}<0.0001)$. Likewise, the mean follicles number per ovary was 2.5 times higher in patients than in controls $(\mathrm{P}<0.0001)$ with and ovarian volume significantly higher in patient than controls ( $\mathrm{P}$ $<0.001$ ) (Table 2).

Table 2: Distribution of the groups according to AMH and findings on echography.

\begin{tabular}{|llll|} 
Parameters & $\begin{array}{l}\mathrm{PCOS}+ \\
(\mathrm{n}=23)\end{array}$ & $\begin{array}{l}\mathrm{PCOS}- \\
(\mathrm{n}=23)\end{array}$ & P value \\
\hline AMH & $6.09 \pm 1.50$ & $3.80 \pm 1.17$ & 0.0001 \\
\hline $\begin{array}{l}\text { AFC } \\
\begin{array}{l}\text { Ovarian } \\
\text { volume }\end{array}\end{array}$ & $15.09 \pm 3.24$ & $6.74 \pm 2.47$ & 0.0001 \\
\hline
\end{tabular}

\section{Correlations}

Age was inversely correlated with $\mathrm{AMH}(\mathrm{r}=-0.301$; $\mathrm{P}$ $<0.05)$ and AFC $(-0.515 ; \mathrm{P}<0.0001)$; suggesting that as age increases, the more AFC and the AMH level decrease. We didn't find a correlation between the AMH and the BMI in the present study (Table 3). AMH was strongly correlated with the AFC $(\mathrm{r}=0.854$; $\mathrm{P}<0.0001)$ suggesting that the AMH level increases with the number of antral follicles. AMH showed a good correlation with the ovarian volume $(\mathrm{r}=0.625 ; \mathrm{P}<0.0001)$ which itself was well correlated with the number of antral follicles.
Table 3: correlations between AMH, age, AFC and ovarian volume.

\begin{tabular}{|llll|} 
Correlations & $\begin{array}{l}\text { Effective } \\
(\mathbf{N}=46)\end{array}$ & $\begin{array}{l}\text { Pearson } \\
\text { coefficient }(\mathbf{r})\end{array}$ & $\begin{array}{l}\text { P value } \\
(\mathbf{P})\end{array}$ \\
\hline Age AMH & 46 & -0.301 & 0.042 \\
\hline Age AFC & 46 & -0.515 & 0.0001 \\
\hline BMI AMH & 46 & -0.050 & 0.741 \\
\hline AFC AMH & 46 & 0.854 & 0.0001 \\
\hline $\begin{array}{l}\text { Ovarian } \\
\text { volume AMH }\end{array}$ & 46 & 0.625 & 0.0001 \\
\hline $\begin{array}{l}\text { Ovarian } \\
\text { volume CFA }\end{array}$ & 46 & 0.678 & 0.0001 \\
\hline
\end{tabular}

Table 4: ROC curve data.

\begin{tabular}{|lcc|}
\hline $\begin{array}{l}\text { AMH threshold level } \\
(\mathrm{ng} / \mathrm{L})\end{array}$ & $\begin{array}{c}\text { Sensitivity } \\
1 \text { - Specificity }\end{array}$ \\
\hline 1.0000 & 1.000 & 1.000 \\
\hline 2.0500 & 1.000 & 0.957 \\
\hline 2.3000 & 1.000 & 0.913 \\
\hline 2.5200 & 1.000 & 0.870 \\
\hline 2.5700 & 1.000 & 0.826 \\
\hline 2.7500 & 1.000 & 0.783 \\
\hline 3.0000 & 1.000 & 0.739 \\
\hline 3.1650 & 1.000 & 0.609 \\
\hline 3.3150 & 1.000 & 0.565 \\
\hline 3.4500 & 1.000 & 0.522 \\
\hline 3.6750 & 1.000 & 0.478 \\
\hline 3.9250 & 0.957 & 0.478 \\
\hline 4.1250 & 0.957 & 0.435 \\
\hline 4.2750 & 0.957 & 0.391 \\
\hline 4.3300 & 0.957 & 0.348 \\
\hline 4.4050 & 0.957 & 0.304 \\
\hline 4.5550 & 0.913 & 0.304 \\
\hline 4.6800 & 0.870 & 0.304 \\
\hline
\end{tabular}

\section{Diagnostic power of AMH in PCOS}

The diagnostic potency of the AMH assay was tested by the ROC procedure.

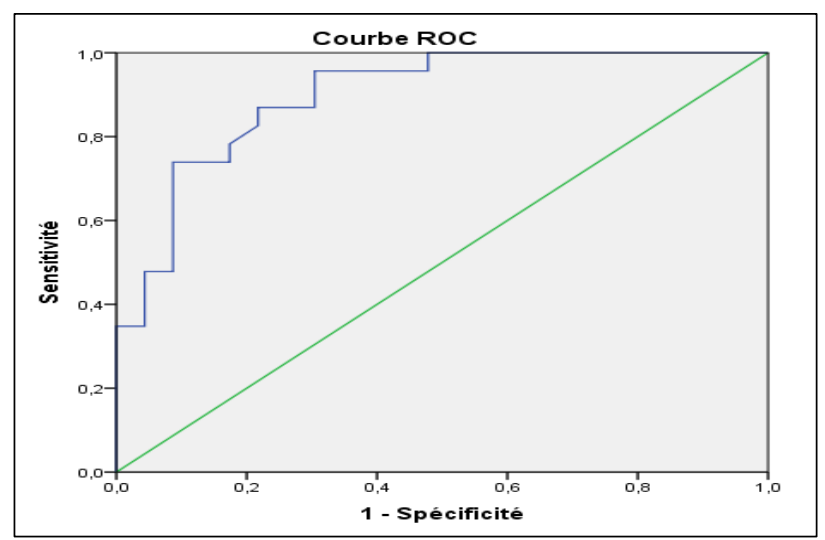

Figure 1: AMH threshold value predictive of PCOS according to ROC curve analysis. 
ROC analysis revealed that the AMH test was very informative for the diagnosis of PCOS, with an area under the curve (AUC) at $0.90(0.81-0.99,95 \% \mathrm{CI})$ according to the parametric method; and the threshold value determined by the intuitive method, given by the farthest point from the diagonal representing the nil contribution test (AUC $=0.5$ ), combining the best compromise sensitivity/specificity was $4.40 \mathrm{ng} / \mathrm{L}$, and predicted PCOS with a sensitivity of $96 \%$ and a specificity of $70 \%$ (Figure 1).

\section{DISCUSSION}

In this study, we investigated whether AMH measurement could be a valuable diagnostic marker of PCOS in an African population for the first time, and its correlation with AFC on endovaginal ultrasound.

We did not find any significant difference between the mean age, weight and BMI of the patients and controls as Homburg et al. ${ }^{9}$ This could be explained by the modest size of our sample. The BMI was not correlated with $\mathrm{AMH}$ as in the study by Cook et al, but the relationship between $\mathrm{AMH}$ and $\mathrm{BMI}$ remains controversial in the literature. ${ }^{10-12}$ Indeed, because obesity is a factor associated with PCOS, obese patients would be expected to have a high AMH level, which is not the case. This is because the pituitary gland of obese patients produces a rapidly metabolized LH isoform that does not optimally act on granulosa to stimulate $\mathrm{AMH}$ production. ${ }^{13}$

The mean value of serum AMH level in the PCOS patient group was almost the double of that of controls $(6.09 \pm 1.80$ versus $3.80 \pm 1.17 ; \mathrm{P}<0.0001)$. Similar data have been found in several studies like that of Tian et al in 2014 (7.4 versus 3.5). ${ }^{14-17}$ This means that even in our black African population, the AMH is significantly higher in PCOS women compared to non-PCOS women. This is due to the numerous follicles whose maturation is blocked in the ovaries of PCOS patients, but also to the deregulation of the expression of the $\mathrm{AMH}$ and of its specific receptor in the presence of $\mathrm{LH}$, responsible of hyperstimulation of AMH production in the presence of $\mathrm{LH}$, and no negative feedback of its receptor supposed to regulate this effect. ${ }^{18}$ The average AFC of PCOS patients was almost two and a half times that of non-PCOS $(15.09 \pm 3.24$ versus $6.74 \pm 2.47 ; \mathrm{P}<0.0001)$ patients due to excessive follicles in their ovaries, which explains why ovarian volume was significantly higher in PCOS compared to controls $(12.02 \pm 3.36$ versus $9.70 \pm 1.41)$.

For correlations, age was inversely correlated with $\mathrm{AMH}$ $(\mathrm{r}=-0.301 ; \mathrm{P}<0.05)$ and CFA $(\mathrm{r}=-0.515 ; \mathrm{P}<0.0001)$, suggesting that as age increases, the AFC and the AMH level decrease. Similar data were found by Ludmila et al and converged with those of the literature which states that $\mathrm{AMH}$ increases at a fairly constant rate up to 25 years, the peak age to become undetectable under 50 years. ${ }^{19}$ The AMH was strongly correlated to the AFC ( $\mathrm{r}$ $=0.854 ; \mathrm{P}<0.0001)$ indicating that the level of $\mathrm{AMH}$ increases with the number of antral follicles. Li et al had similar results and this is due to the fact that, AMH is produced in the granulosa of the ovarian follicles. ${ }^{20}$ The AMH showed a good correlation with the ovarian volume ( $\mathrm{r}=0.625 ; \mathrm{P}<0.0001)$ which itself was well correlated to the AFC ( $\mathrm{r}=0.678 ; \mathrm{P}<0.0001)$. Dolfing et al had similar results and this would be due to the fact that, the ovarian volume is proportional to the number of follicles which determine the AMH level.

In this study, the diagnostic performance of $\mathrm{AMH}$ was assessed using the ROC curves. ROC analysis revealed that the AMH test was very informative for the diagnosis of PCOS, with a satisfying area under the curve (AUC) of serum AMH assay at $0.90(0.81-0.99,95 \% \mathrm{CI})$ and the threshold value, given by the farthest point from the diagonal representing the nil contribution test $(\mathrm{AUC}=$ 0.5), combining the best compromise sensitivity/specificity was $4.40 \mathrm{ng} / \mathrm{L}$, and predicted PCOS with a sensitivity of $96 \%$ and a specificity of $70 \%$ (Figure $1)$.

The threshold value thus obtained had excellent sensitivity and satisfactory specificity, making AMH a predictive marker in PCOS. Dewailly et al had similar results, and suggested that a cut off of $4.9 \mathrm{ng} / \mathrm{L}$ could replace polycystic ovarian morphology on endovaginal ultrasound with a sensitivity of $92 \%$ and a specificity of $97 \% .{ }^{21}$ Casadei et al had an AMH threshold value of 4.62 $\mathrm{ng} / \mathrm{L}$ with a sensitivity and specificity of $95 \% .^{22}$ Iliodromiti et al in their meta-analysis of 10 studies found a cut off of $4.7 \mathrm{ng} / \mathrm{L}$ with a sensitivity of $82 \%$ and a specificity of $79.4 \% .{ }^{16}$ The difference found in these studies could be explained by the fact that our study population consisted of black African subjects, or due to the size of the studies samples and the criteria used to define PCOS. AMH can therefore be used as a diagnostic marker for PCOS, however, due to the strong correlation observed with the CFA, it would be appropriate to perform an endovaginal ultrasound to improve its specificity.

\section{CONCLUSION}

In conclusion, AMH is a predictive marker for PCOS. It is highly correlated with AFC and ovarian volume and appears to decrease with age. It offers good diagnostic performance in PCOS, with a threshold value of 4.40 $\mathrm{ng} / \mathrm{L}$ for a sensitivity of $96 \%$ and specificity of $70 \%$.

Funding: No funding sources

Conflict of interest: None declared

Ethical approval: The study was approved by the Institutional Ethics Committee

\section{REFERENCES}

1. Norman RJ, Dewailly D, Legro RS, Hickey TE. Polycystic ovary syndrome. The Lancet. 2007;370(9588):685-97. 
2. Stein IF, Leventhal ML. Amenorrhea associated with bilateral polycystic ovaries. $1935 ; 29(2): 181-91$.

3. Rotterdam ESHRE/ASRM-sponsored PCOS consensus workshop group. Revised 2003 consensus on diagnostic criteria and long-term health risks related to polycystic ovary syndrome. Fertil Steril. 2004;81(1):19-25.

4. Johnson TRB, Kaplan LK, Ouyang P, Rizza RA, Health NI. Evidence-based methodology workshop on polycystic ovary syndrome. Bethesda, Maryland: National Institutes of Health. 2012.

5. Azziz R, Carmina E, Dewailly D, DiamantiKandarakis E, Escobar-Morreale HF, Futterweit W, et al. The Androgen excess and PCOS society criteria for the polycystic ovary syndrome: the complete task force report. Fertil Steril. 2009;91(2):456-88.

6. Knochenhauer ES, Key TJ, Kahsar-Miller M, Waggoner W, Boots LR, Azziz R. Prevalence of the polycystic ovary syndrome in unselected black and White women of the Southeastern United States: a prospective study. J Clin Endocrinol Metab. 1998;83(9):3078-82.

7. Tehrani FR, Simbar M, Tohidi M, Hosseinpanah F, Azizi F. The prevalence of polycystic ovary syndrome in a community sample of Iranian population: Iranian PCOS prevalence study. Reprod Biol Endocrinol. 2011;9:39.

8. Chao KC, Ho CH, Shyong WY, Huang CY, Tsai SC, Cheng HY, et al. Anti-Mullerian hormone serum level as a predictive marker of ovarian function in Taiwanese women. J Chin Med Assoc. 2012;75(2):70-4.

9. Homburg R, Ray A, Bhide P, Gudi A, Shah A, Timms P, et al. The relationship of serum antiMullerian hormone with polycystic ovarian morphology and polycystic ovary syndrome: a prospective cohort study. Hum Reprod. 2013;28(4):1077-83.

10. Cook CL, Siow Y, Brenner AG, Fallat ME. Relationship between serum Müllerian-inhibiting substance and other reproductive hormones in untreated women with polycystic ovary syndrome and normal women. Fertil Steril. 2002;77(1):141-6.

11. Freeman EW, Gracia CR, Sammel MD, Lin H, Lim LCL, Strauss JF. Association of anti-mullerian hormone levels with obesity in late reproductive-age women. Fertil Steril. 2007;87(1):101-6.

12. Dolfing J, Schweitzer D, Wolffenbuttel B. Metabolic and hormonal parameters in lean PCOS women. 2012.

13. Taylor AE, McCourt B, Martin KA, Anderson EJ, Adams JM, Schoenfeld D, et al. Determinants of abnormal gonadotropin secretion in clinically defined women with polycystic ovary syndrome. J Clin Endocrinol Metab.1997;82(7):2248-56.
14. Tian X, Ruan X, Mueck AO, Wallwiener D, Wang J, Liu S, et al. Serum anti-Müllerian hormone and insulin resistance in the main phenotypes of nonobese polycystic ovarian syndrome women in China. Gynecol Endocrinol. 2014;30(11):836-9.

15. Sahmay S, Atakul N, Aydogan B, Aydın Y, Imamoglu M, Seyisoglu H. Elevated serum levels of anti-Müllerian hormone can be introduced as a new diagnostic marker for polycystic ovary syndrome. Acta Obstet Gynecol Scand. 2013;92(12):1369-74.

16. Iliodromiti S, Kelsey TW, Anderson RA, Nelson SM. Can anti-Müllerian hormone predict the diagnosis of polycystic ovary syndrome? a systematic review and meta-analysis of extracted data. J Clin Endocrinol Metab. 2013;98(8):3332-40.

17. Fallat ME, Siow Y, Marra M, Cook C, Carrillo A. Müllerian-inhibiting substance in follicular fluid and serum: a comparison of patients with tubal factor infertility, polycystic ovary syndrome, and endometriosis. Fertil Steril. 1997;67(5):962-5.

18. Pierre A, Peigné M, Grynberg M, Arouche N, Taieb $\mathrm{J}$, Hesters L, et al. Loss of LH-induced downregulation of anti-Müllerian hormone receptor expression may contribute to anovulation in women with polycystic ovary syndrome. Hum Reprod. 2013;28(3):762-9.

19. Barbakadze L, Kristesashvili J, Khonelidze N, Tsagareishvili G. The correlations of anti-mullerian hormone, follicle-stimulating hormone and antral follicle count in different age groups of infertile women. Int J Fertil Steril. 2015;8(4):393-8.

20. Li R, Gong F, Zhu Y, Fang W, Yang J, Liu J, et al. Anti-Müllerian hormone for prediction of ovarian response in Chinese infertile women undergoing IVF/ICSI cycles: a prospective, multi-centre, observational study. Reprod Biomed Online. 2016;33(4):506-12.

21. Dewailly D, Lujan ME, Carmina E, Cedars MI, Laven J, Norman RJ, et al. Definition and significance of polycystic ovarian morphology: a task force report from the Androgen excess and polycystic ovary syndrome society. Hum Reprod. 2014;20(3):334-52.

22. Casadei L, Madrigale A, Puca F, Manicuti C, Emidi E, Piccione E, et al. The role of serum anti-Müllerian hormone $(\mathrm{AMH})$ in the hormonal diagnosis of polycystic ovary syndrome. Gynecol Endocrinol. 2013;29(6):545-50.

Cite this article as: Ndoua NCC, Ngah AG, Koh MV, Belinga E, Kemfang JD, Kasia JM. Correlation between the anti-Müllerian hormone and endovaginal ultrasound in the predictivity of polycystic ovary syndrome at Chracerh. Int J Reprod Contracept Obstet Gynecol 2017;6:5183-7. 